\title{
EL AGROTURISMO COMO DESARROLLO DE TURISMO RURAL
}

Ing. Mg. Eufemia Ramos ${ }^{1}$ Dra. Carrasco Ruano Tatiana ${ }^{2}$, M.Sc Efraín Velasteguí Lòpez. ${ }^{3}$ \& Tania Cristina Cevallos Punguil. ${ }^{4}$

\begin{abstract}
Agrotourism is the sustainable use of natural and cultural resources preserving the biodiversity of a sector whose purpose is to attract tourists with cultivated landscapes. Rural sectors implementing this activity will achieve economic development to improve the quality of life of farmers. The crops will be organic, efficient, profitable and competitive.

Agrotourism ventures are family-run with few workers, low training options and limited economic and financial resources. The characteristics of the field work are specific and require constancy and experience. Daily life in the rural sector is characterized by traditions, customs, political differences, lack of security in the achievements, lack of time, low profitability. In addition, the state's dependence on the development of basic services and the lack of a socio-productive vision limits success and does not autonomously solve the needs of the population.

The climate of Canton Cevallos and geography allows the cultivation of vegetables, legumes, aromatic species, medicinal and fruit species; The same that will have paths for tourists to circulate without affecting crops. The basic services and security in the hotels houses belonging to the owners of the farms and plots will be basic so that this experience of the tourists is unforgettable and will offer things to see and things to do to meet expectations and enjoyment.

The research will be delivered to the Decentralized Autonomous Government of the Cevallos Canton to motivate the local entrepreneurs to carry out their new business with strengthened bases in the planned design to offer agrotourism and to market based on training, technical assistance and support of the financial entities for the beginning and Business development. Community education is important to take advantage of the infrastructure and participation of productive sectors such as: footwear, organic agriculture, fruit, beekeeping, poultry, livestock, gastronomy, livestock tourism, among others; These will be integrated in an orderly and planned manner so as not to carry out isolated efforts and obtain a differentiated position because it is a strategic place due to the climatic characteristics and to be part of the circuit of the Inter-Andean Cruise Train.
\end{abstract}

Keywords: Agrotourism, Rural Tourism, Enterprising, Cultivated Landscapes

CÓDIGO UNESCO: Administración 590900

\footnotetext{
${ }^{1}$ Universidad Técnica de Ambato, Ambato, Ecuador, a.ramos@uta.edu.ec

${ }^{2}$ Universidad UNIR”, España , Madrid, tcarrascor@yahoo.com

${ }^{3}$ Universidad Técnica de Ambato, Ambato, Ecuador, le.velastegui@uta.edu.ec

${ }^{4}$ Universidad Estatal Amazónica. Puyo, Ecuador. tc.cevallosp@uea.edu.ec
} 


\section{RESUMEN}

El agroturismo es el aprovechamiento sustentable de los recursos naturales y culturales preservando la biodiversidad de un sector cuyo propósito es atraer turistas con paisajes cultivados. Los sectores rurales que implementen esta actividad lograrán obtener desarrollo económico para mejorar la calidad de vida de los agricultores. Los cultivos serán orgánicos, eficientes, rentables y competitivos.

Los emprendimientos de agroturismo son de carácter familiar con pocos trabajadores, bajas opciones de capacitación y limitados recursos económicos y financieros. Las características del trabajo de campo son específicas y requieren de constancia y experiencia. La vida diaria en el sector rural se caracteriza por tradiciones, costumbres, diferencias políticas, falta de seguridad en los logros, falta de tiempo, baja rentabilidad. Además, la dependencia estatal para el desarrollo de servicios básicos y la carencia de una visión socioproductiva limita al éxito y no solucionan con autonomía las necesidades de la población.

El clima del Cantón Cevallos y la geografía permite el cultivo de hortalizas, legumbres, especies aromáticas, especies medicinales y frutales; los mismos que contarán con senderos para que circulen los turistas sin afectar los cultivos. Los servicios básicos y seguridad en las casas hoteles que pertenecen a los dueños de las fincas y parcelas será básico para que esta experiencia de los turistas sea inolvidable y se ofrecerá cosas qué ver y cosas qué hacer para logar satisfacer las expectativas y el disfrute.

Se entregará la investigación al Gobierno Autónomo Descentralizado del Cantón Cevallos para motivar a los emprendedores locales realicen sus nuevos negocios con bases fortalecidas en el diseño planificado para ofrecer agroturismo y comercializar en base a capacitación, asistencia técnica y apoyo de las entidades financieras para el inicio y desarrollo del negocio.

La educación a la comunidad es importante para aprovechar la infraestructura y la participación de los sectores productivos como: calzado, agricultura orgánica, frutícola, apícola, avícola, ganadero, gastronomía, turístico pecuario, entre otras; éstos se integrarán de manera ordenada y planificada para no ejecutar esfuerzos aislados y obtener un posicionamiento diferenciado por tratarse de un lugar estratégico por las características climáticas y por formar parte del circuito del Tren Crucero Interandino.

Palabras claves: Agroturismo, turismo rural, emprendedor, paisajes cultivados

\section{INTRODUCCIÓN}

Las empresas de agroturismo son de carácter familiar con pocos trabajadores, bajas opciones de capacitación y limitados recursos económicos y financieros. Las características del trabajo de campo son específicas y requieren de constancia y experiencia. La vida diaria en el sector rural se caracteriza por tradiciones, costumbres, diferencias políticas, falta de seguridad en los logros, falta de tiempo, baja rentabilidad. Además, la dependencia estatal para el desarrollo de servicios básicos y la carencia de una visión socio productiva limita al éxito y no solucionan con autonomía las necesidades de la población. 
El desarrollo del Cantón Cevallos está vinculado al modelo de desarrollo turístico bajo esta estructura se requiere que las familias que se dedican a la agricultura tradicional diversifiquen la producción y que se trabaje con una visión de oferta turística como una estrategia de desarrollo económico y que la crianza de animales menores también se oferte a los visitantes.

Schaerer y Sirven (como se citó en Inostroza, 2008) piensa que los agricultores atraviesan por serios problemas que dificultan su buen vivir por no contar con asistencia técnica para las actividades turísticas y las políticas públicas son insuficientes para la promoción. Los operadores de turismo no promueven, ni venden productos de turismo rural; por tanto, se requiere ofrecer estrategias y alternativas con apoyo del sector público para mejorar la economía de la comunidad rural que menor oportunidad tiene a su alcance.

Las comunidades que identifiquen y valoren los recursos que poseen, podrán desarrollar una actividad de turismo a futuro; puesto que, los principales atributos que un visitante desea conocer es un medio natural bien conservado y con señalética, en un contexto natural único y diferente. La naturaleza y la cultura son parte de la actividad turística (Inostroza, 2008).

Los desarrollos económicos locales exigen la confianza entre actores para trabajar en forma conjunta; esto requiere de la coordinación y articulación entre los sectores público y privado y el criterio de emprendimientos socio productivos que genere desarrollo turístico de territorio, en donde cada sector y actor turístico tiene que cumplir su rol y conocer que es necesario e importante aprovechar los recursos que posee para el desarrollo del turismo como un negocio rentable y de desarrollo (Alburquerque, 2004).

\section{ANTECEDENTES}

El Ecuador es un país que posee ecosistemas biodiversos y cuenta con las condiciones óptimas para desarrollar actividades turísticas como: turismo de descanso o simplemente sol y playa, turismo cultural, ecoturismo, turismo de aventura, turismo religioso, turismo gastronómico, turismo comunitario y agroturismo.

El proyecto de Ley Orgánica de la Libre Competencia Económica asume el reto de contribuir al desarrollo económico del Ecuador, dentro de la economía social de Ecuador, dentro de la economía social de mercado, a través de la promoción de una competencia sana entre los agentes económicos, como herramienta que contribuirá sobre llevar la globalización y la liberación comercial ( Dirección Nacional del Comercio Exterior, 1999).

La Constitución vigente determina que el Estado debe promover el desarrollo de actividades y mercados de actividades y mercados competitivos, a través de la promoción de la libre competencia; sancionar prácticas monopólicas y otras que impidan o la distorsionen, todo esto en defensa del bien común (Dirección Nacional del Comercio Exterior, 1999).

"A nivel hemisférico, solamente 14 países disponen de Autoridades y Leyes de Competencia. Ecuador y Bolivia en el marco de la CAN no cuentan con Autoridad y Ley en esta materia" (Dirección Nacional del Comercio Exterior, 1999). 
La Ley de Turismo regula a todos quienes ejercen la actividad turística, puesto que en el Art. 12 manifiesta que: "Cuando las comunidades locales organizadas y capacitadas deseen prestar servicios turísticos, recibirán del Ministerio de Turismo o sus Delegados, en igualdad de condiciones todas las facilidades necesarias para el desarrollo de estas actividades, las que no tendrán exclusividad de operación en el lugar en que se presten sus servicios y se sujetarán a lo dispuesto en ésta Ley y a los Reglamentos respectivos" (Art.12, Ley de Turismo, 2002).

La Organización Mundial del Turismo 2007 define que "el turismo es un fenómeno social, cultural y económico relacionado con el movimiento de las personas a lugares que se encuentran fuera de su lugar de residencia habitual por motivos personales o de negocios/profesionales" (Asamblea General, 2003).

El agroturismo es un segmento del turismo rural con la particularidad de que se ubica en granjas y que tiene la capacidad de hacer partícipe al visitante o turista en las diferentes actividades agropecuarias y que el propósito es brindar diversas opciones de distracción y atraer turistas que amen la naturaleza y principalmente el paisaje cultivado. Para ofrecer agroturismo es necesario desarrollar cultivos orgánicos eficientes, rentables, competitivos en base a una planificación y organización son señalética y senderos específicos que ofrezca valor agregado a lo cotidiano (Valenzuela, 2007).

Cevallos está ubicado en el sector centro-sur de la Provincia de Tungurahua y cuenta con la infraestructura turística básica por formar parte del Tren Crucero Interandino por lo que se propone vincular el agroturismo a los demás sectores productivos y turísticos de la zona y mejorar la economía de la población rural (Ganán, 2015).

Si la oferta de agroturismo atrae a los turistas aumentará los ingresos de la población rural siempre hay que conservar la realidad natural. Además, se protegerá el medio ambiente y se ofrecerá la infraestructura turística de los otros sectores productivos; puesto que el agroturismo será una actividad asociada a los otros servicios turísticos (Crosby, 2009).

(Montaner, 1998) en el diccionario de turismo indica que el agroturismo "se aleja mucho de los métodos estandarizados que caracterizan el turismo de masas y que en cierta medida son una traslación de los hábitos urbanos al centro turístico".

"El cantón Cevallos está ubicado en el sector centro-sur de la Provincia de Tungurahua. Esta jurisdicción está limitada al norte con Ambato, al sur con Mocha y Quero, al este con Tisaleo y al oeste con Pelileo" (Pilar, 2011).

En base a la información de la Agendas para la Transformación Productiva Territorial de Tungurahua, este cantón está a $15 \mathrm{~km}$ del sur-oriente del cantón Ambato y tiene un clima especial lo que favorece a los cultivos y fruticultura con muchas variedades de: claudia, manzana, fresa, capulí, pera, durazno y flores. Los poblados más importantes son: Santa Rosa, San Pedro, La Floresta, Jesús del Gran Poder, El Belén, El Mirador, Santo Domingo, Tambo, Tamboloma, Andignato, Aire Libre y Agua Santa. Entre los atractivos turísticos están los monumentos a: Pedro Fermín Cevallos, Ferroviario, Artesano, La Madre, La Estación del Ferrocarril; además, la iglesia, el sendero Pachanlica y la cascada de Jun Jun. La población es de 6.873 de acuerdo a cifras del Instituto Nacional de Estadísticas y Censos. Sobresale en la producción de calzado y manufactura de microempresas textiles. En la 
infraestructura turística cuenta con las hosterías La Bonita y Pueblito Serrano y los hoteles reciben a los visitantes con todos los servicios complementarios como: piscina, restaurante, spa, zonas verdes. Los platos típicos que se pueden degustar son hornado, fritada, yahuarlocro, carnes, caldo de gallina, cuy, conejo, pollo, chicha y platos a la carta en los paraderos Las Rosas, Rincón Cevallense, Súper Cuy, Doña Rosita, El Cajón entre otros. Las vías de acceso son varias y todas de primer orden con la señalética e información de cada comunidad (Chiriboga, 2011).

En la zona se implementaron varios programas de reactivación productiva por la afectación del proceso de activación del volcán Tungurahua por lo que realiza esfuerzos para mejor la calidad de vida de los habitantes.

El clima templado del Cantón Cevallos favorece el cultivo de hortalizas, legumbres, especies aromáticas, especies medicinales y frutales. Existen fincas con servicios básicos y seguridad para operar como casas hoteles; puesto que, son muy cercanas a la estación del ferrocarril y además cuenta con vías de primer orden.

Además, cuenta con Escuelas, Colegios, Facultad de Ingeniería Agronómica, canal de riego HuachiPelileo, agua potable, luz, alcantarillado sanitario, cooperativas de ahorro y crédito, cooperativas de transporte, asfaltado, mercados, coliseo, estadios.

En 1092 se construyó la Estación del Ferrocarril en esta población y se reactiva en el 2013 con el Tren Crucero Interandino; por tanto, este cantón es una parada incluida para el turismo.

El cultivo de hortalizas, legumbres, especies aromáticas, especies medicinales y frutales contarán con senderos para que circulen los turistas sin afectar los cultivos, servicios básicos y seguridad en las casas hoteles para que esta experiencia sea inolvidable se debe ofrecer cosas qué ver y cosas qué hacer (Alvarez, 2015).

En base a la Agenda de Políticas Públicas 2035 y con el fin de unificar la gestión en la Provincia de Tungurahua se involucró a la comunidad a ratificar la prioridad del desarrollo rural con acciones de participación ciudadana y generar estrategias en turismo como una parte de los acuerdos y planteamientos en la propuesta de cambio de la matriz productiva y se generó el Comité Provincial de Turismo para promocionar las rutas turísticas y dinamizar el trabajo rural agrícola (UTA, 2016).

(Marreno, 2014) manifiesta que "la competitividad no puede concebirse sin una base sostenible y desarrollo del capital humano".

Los emprendimientos de agroturismo son de carácter familiar con pocos trabajadores, bajas opciones de capacitación y limitados recursos económicos y financieros. Las características del trabajo de campo son específicas y requieren de constancia y experiencia. La vida diaria en el sector rural se caracteriza por tradiciones, costumbres, diferencias políticas, falta de seguridad en los logros, falta de tiempo, baja rentabilidad. Además, la dependencia estatal para el desarrollo de servicios básicos y la carencia de una visión socio-productiva limita al éxito y no solucionan con autonomía las necesidades de la población (Recalde, 2010).

El clima del Cantón Cevallos y la geografía permite el cultivo de hortalizas, legumbres, especies aromáticas, especies medicinales y frutales; los mismos que contarán con senderos para que circulen 
los turistas sin afectar los cultivos. Los servicios básicos y seguridad en las casas hoteles que pertenecen a los dueños de las fincas y parcelas será básico para que esta experiencia de los turistas sea inolvidable y se ofrecerá cosas qué ver y cosas qué hacer para logar satisfacer las expectativas y el disfrute (Rosario, 2011).

Se entregará la investigación al Gobierno Autónomo Descentralizado del Cantón Cevallos para motivar a los emprendedores locales realicen sus nuevos negocios con bases fortalecidas en el diseño planificado para ofrecer agroturismo y comercializar en base a capacitación, asistencia técnica y apoyo de las entidades financieras para el inicio y desarrollo del negocio.

La educación a la comunidad es importante para aprovechar la infraestructura y la participación de los sectores productivos como: calzado, agricultura orgánica, frutícola, apícola, avícola, ganadero, gastronomía, turístico pecuario, entre otras; éstos se integrarán de manera ordenada y planificada para no ejecutar esfuerzos aislados y obtener un posicionamiento diferenciado por tratarse de un lugar estratégico por las características climáticas y por formar parte del circuito del Tren Crucero Interandino (Cevallos, 2015).

\section{METODOLOGÍA DE LA INVESTIGACIÓN.}

El estudio es descriptivo y se basa en características del turismo rural bajo el método de investigaciónacción puesto que se desarrolló en el entorno del objeto de estudio y realizamos el análisis de las variables.

El propósito es aprovechar la infraestructura y la participación de los sectores productivos como: calzado, agricultura orgánica, frutícola, apícola, avícola, ganadero, gastronomía, turístico pecuario, entre otras e integrar de manera ordenada y planificada para no ejecutar esfuerzos aislados para obtener un posicionamiento diferenciado por tratarse de un lugar estratégico por las características climáticas y por formar parte del circuito del Tren Crucero Interandino. Al buscar este tipo de estrategias de inserción, promoción y comercialización del agroturismo fomentaremos la preservación de la identidad cultural y el turismo rural basado el "La Declaración de San José sobre Turismo Rural Comunitario", que manifiesta que es un componente estratégico de desarrollo local, nacional y regional por la generación de beneficios económicos, empleo y bienestar de las comunidades anfitrionas (Sán José, 2003)

Casi todas las localidades rurales tienen algún atractivo turístico sin dejar de lado las labores del campo, ni desplazar de los trabajos cotidianos por lo que siempre existe tendencia de turistas que se interesan por la vida rural y vincula la vida urbano-rural y para ello deberá efectuarse un estudio de oferta-demanda, competencia, tendencia de mercado y el impacto orientado a la gestión de la calidad de los servicios que conlleven al desarrollo sostenido de los residentes rurales (Zamora, 2016).

Se seleccionó como instrumento de investigación la entrevista para contar con información sobre las características del turismo rural y determinar las fortalezas y debilidades de los habitantes para buscar la alternativa económica real y determinar cuántas familias ofrecerían servicios de agroturismo con casas hoteles para turistas urbanos. 
Población y muestra.

Las familias orientan su trabajo a metas individuales y no se planean objetivos para generar ingresos económicos complementarios para sus hogares; por tanto, como estrategia

\section{Plan de Procesamiento y Análisis.}

El Ecuador y todas las zonas geográficas de la región sierra tienen atractivos turísticos y posee las condiciones para implementar el agroturismo como alternativa de emprendimiento para mejorar la calidad de vida de los habitantes de cada comunidad. Estos emprendimientos son familiares con pocos trabajadores, bajas opciones de capacitación y limitados recursos económicos y financieros. Las características del trabajo de campo son específicas y requieren de constancia y experiencia. La vida diaria en el sector rural se caracteriza por tradiciones, costumbres, diferencias políticas, falta de seguridad en los logros, falta de tiempo, baja rentabilidad. Además, la dependencia estatal para el desarrollo de servicios básicos y la carencia de una visión socio-productiva limita al éxito y no proponen soluciones con autonomía para cumplir las necesidades de la población.

Las políticas públicas del 2035 tienen la capacidad de definir metas colectivas de los sectores productivos y de movilizar recursos para los resultados de desarrollo planificado por tanto, el sector público requiere incorporar al sector privado en este proceso que apuntan a objetivos sociales y políticos que satisface los derechos de todo ciudadano y cumplir con los requerimientos del Plan Nacional del Buen Vivir 2013-2017, el mismo que ofrece alternativas de una sociedad más justa y de inclusión para todos los sectores de la población en la actividad turística (UTA, 2016).

\section{EDADES DE CLIENTES NACIONALES Y EXTRANJEROS DEL TREN CRUCERO}

Tabla 1 Edades

\begin{tabular}{ccc}
\hline $\begin{array}{c}\text { Edad } \\
\text { (años) }\end{array}$ & $\begin{array}{c}\text { Clientes (Hombres, } \\
\text { mujeres) }\end{array}$ & $\begin{array}{c}\text { Frecuencia } \\
\text { relativa }\end{array}$ \\
\hline $\begin{array}{c}\text { Menor de } \\
25\end{array}$ & 27 & $10 \%$ \\
$25-30$ & 13 & $5 \%$ \\
$30-35$ & 62 & $22 \%$ \\
$35-40$ & 59 & $21 \%$ \\
$40-50$ & 46 & $16 \%$ \\
50 y & 73 & $26 \%$ \\
mayor & & $\mathbf{1 0 0 \%}$ \\
TOTAL & $\mathbf{2 8 0}$ & \\
\hline
\end{tabular}

Elaborado por: Grupo Investigador

Fuente: Turismo ferrocarril Tren Crucero, Cantón Cevallos

Figura 1 Edades 


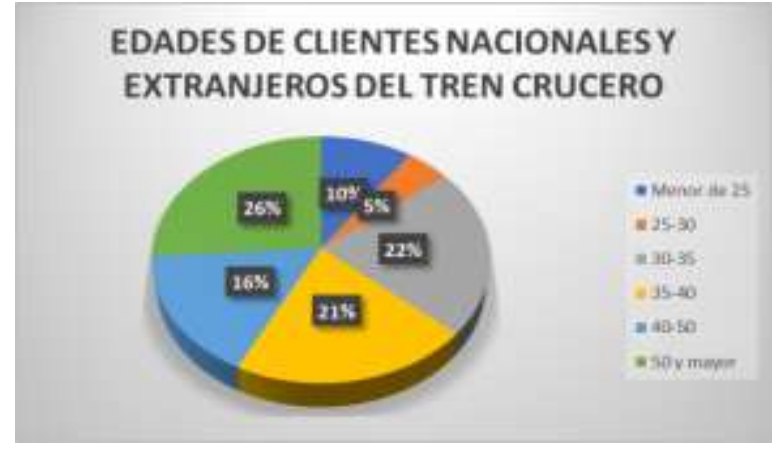

Elaborado por: Grupo investigador.

Fuente: Turismo ferrocarril Tren Crucero, Cantón Cevallos

\section{PROCEDENCIA DE LOS CLIENTES NACIONALES DEL TREN CRUCERO}

Tabla 2 Procedencia

\begin{tabular}{lll}
\hline Guayaquil & $\mathbf{6 7}$ & $\mathbf{2 7 \%}$ \\
\hline Quito & 78 & $31 \%$ \\
Cuenca & 49 & $20 \%$ \\
Ambato & 42 & $17 \%$ \\
Puyo & 12 & $5 \%$ \\
TOTAL & $\mathbf{2 8 0}$ & $\mathbf{1 0 0 \%}$ \\
\hline
\end{tabular}

Fuente: Turismo ferrocarril Tren Crucero, Cantón Cevallos.

Elaborado: Grupo Investigador.

\section{Figura 2 Procedencia}

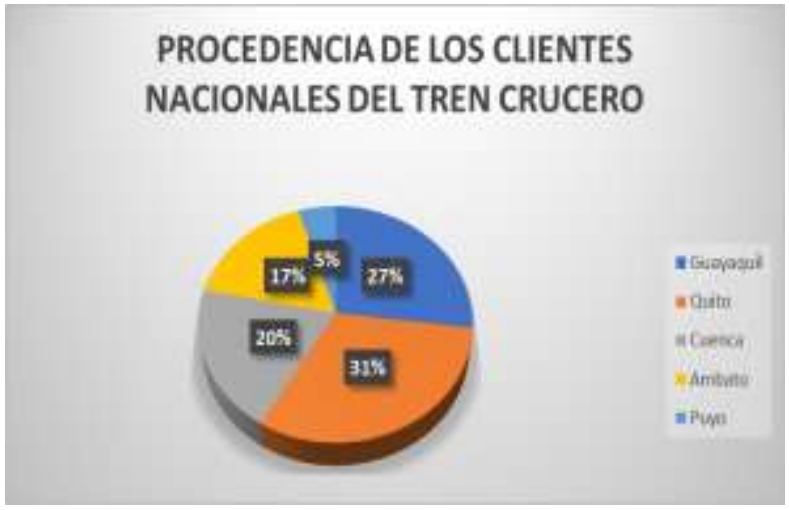

Elaborado por: Grupo Investigador

$\mathrm{N}^{\circ}$ de fincas y casas de parcelas que pueden acoger turistas para casa-hotel.

Tabla 3 


\begin{tabular}{ccc}
\hline $\begin{array}{c}\text { NIVEL DE } \\
\text { INFRAESTRUCTURA }\end{array}$ & $\begin{array}{c}\text { FRECUENCIA } \\
\text { ABSOLUTA }\end{array}$ & $\begin{array}{c}\text { FRECUENCIA } \\
\text { RELATIVA }\end{array}$ \\
\hline Alto & 10 & 11 \\
Medio & 47 & 53 \\
Bajo & 32 & 36 \\
& $\mathbf{8 9}$ & $\mathbf{1 0 0}$
\end{tabular}

Elaborado por: Grupo Investigador

Figura 3

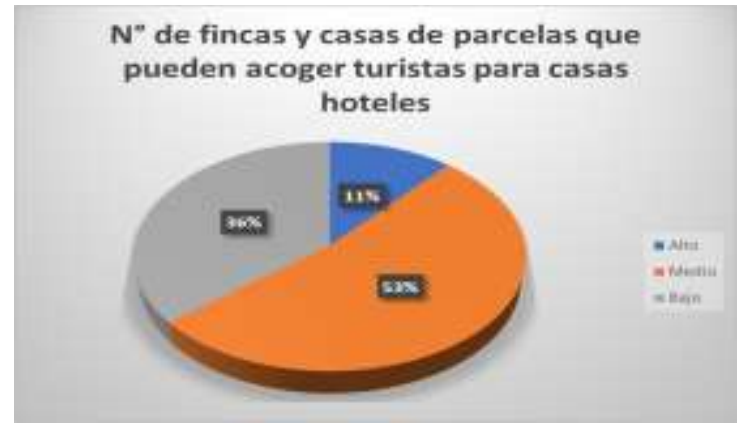

Elaborado por: Grupo Investigador

PREFERENCIA POR ACTIVIDADES TURÍSTICAS A REALIZAR

Tabla 4 Preferencia

\begin{tabular}{ccccc}
\hline ACTIVIDAD & $\begin{array}{c}\text { FRECUENCIA } \\
\text { ABSOLUTA }\end{array}$ & $\begin{array}{c}\text { FRECUENCIA } \\
\text { ABSOLUTA } \\
\text { ACUMULADA }\end{array}$ & $\begin{array}{c}\text { FRECUENCIA } \\
\text { RELATIVA }\end{array}$ & $\begin{array}{c}\text { FRECUENCIA } \\
\text { RELATIVA } \\
\text { ACUMULADA }\end{array}$ \\
\hline Cabalgata & 20 & 20 & 7 & 7 \\
Senderismo & 24 & 44 & 9 & 16 \\
Biking & 28 & 72 & 10 & 26 \\
Camping & 18 & 90 & 6 & 32 \\
Turismo & 25 & 115 & 9 & 41 \\
Vivencial & & 131 & 6 & 47 \\
$\begin{array}{c}\text { Turismo } \\
\text { Comunitario }\end{array}$ & 16 & 158 & 10 & 57 \\
$\begin{array}{c}\text { Ecoturismo } \\
\text { Agroturismo }\end{array}$ & 27 & 197 & 14 & 71 \\
$\begin{array}{c}\text { Fotografía } \\
\text { turística }\end{array}$ & 28 & 225 & 10 & 81 \\
Recorrido en & 28 & & 9 & 90 \\
granjas & 26 & 251 & 10 & 100 \\
$\begin{array}{c}\text { Turismo } \\
\text { cultural }\end{array}$ & 29 & 280 & $\mathbf{1 0 0}$ & \\
TOTAL & $\mathbf{2 8 0}$ & & & \\
\hline
\end{tabular}

Elaborado por: Grupo Investigador

Figura 4 Preferencias 


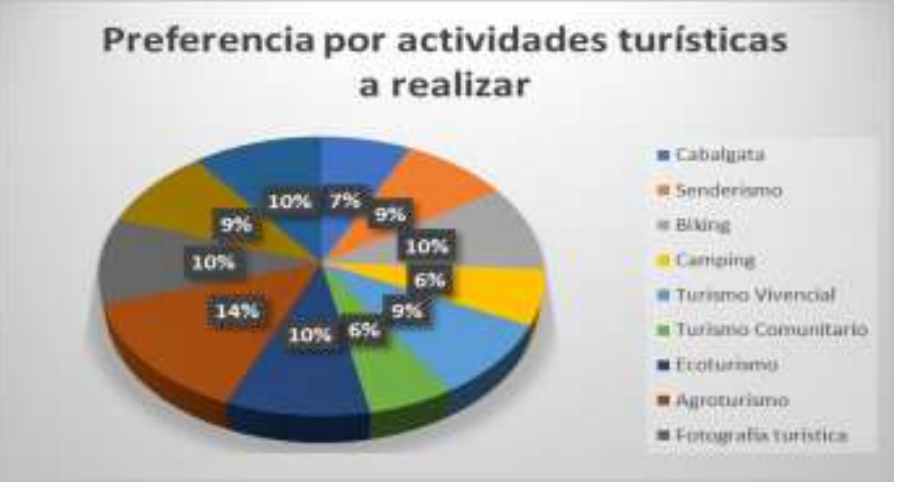

Elaborado por: Grupo Investigador

\section{PROMEDIO DE ESTANCIA}

Tabla 5 Promedio de Estancia

\begin{tabular}{ccccc}
\hline $\mathrm{N}^{\circ}$ DÍAS & $\begin{array}{c}\text { FRECUENCIA } \\
\text { ABSOLUTA }\end{array}$ & $\begin{array}{c}\text { FRECUENCIA } \\
\text { ABSOLUTA } \\
\text { ACUMULADA }\end{array}$ & $\begin{array}{c}\text { FRECUENCIA } \\
\text { RELATIVA }\end{array}$ & $\begin{array}{c}\text { FRECUENCIA } \\
\text { RELATIVA } \\
\text { ACUMULADA }\end{array}$ \\
\hline 1 día & 150 & 150 & 54 & 54 \\
2 días & 80 & 230 & 29 & 83 \\
3 días & 50 & 280 & 17 & 100 \\
Total & $\mathbf{2 8 0}$ & & $\mathbf{1 0 0}$ & \\
\hline
\end{tabular}

Elaborado por: Grupo Investigador

Figura 5 Promedio de Estancia

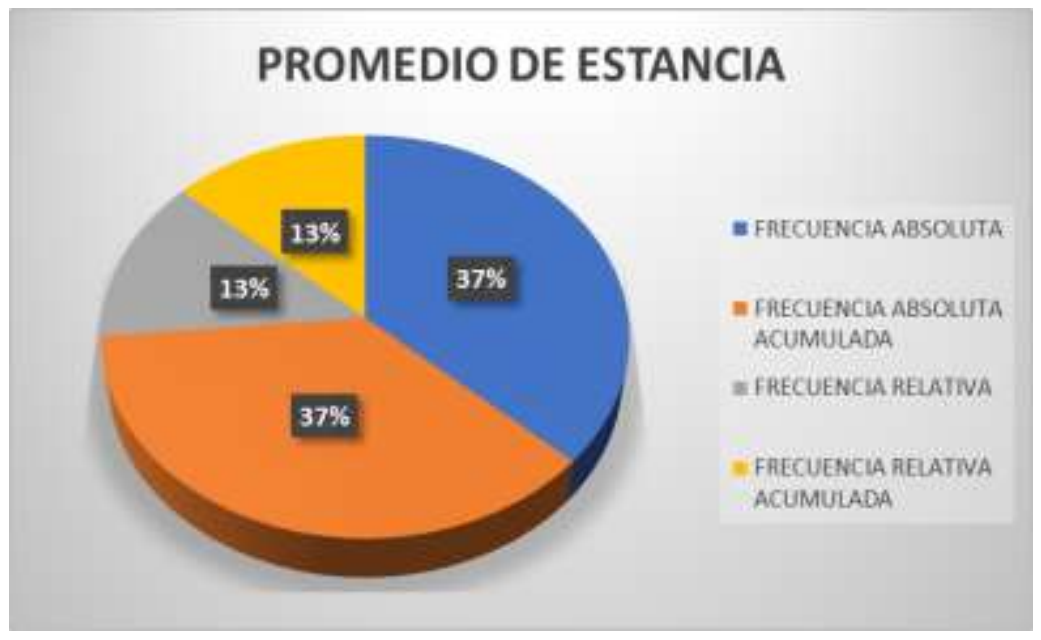

Elaborado por: Grupo Investigador

El clima del Cantón Cevallos y la geografía permite el cultivo de hortalizas, legumbres, especies aromáticas, especies medicinales y frutales; los mismos que no cuentan con senderos para que 
circulen los turistas sin afectar los cultivos. Esta población tiene servicios básicos y se recomienda que se ofrezca seguridad. Como alternativa de emprendimiento es que los agricultores de granjas y parcelas ofrezcan a los turistas una habitación con comodidades básicas y se conviertan en casas hoteles para que la experiencia de los turistas sea inolvidable y experiencia de los turistas sea inolvidable y se ofrezca cosas qué ver y cosas qué hacer para logar satisfacer las expectativas y el disfrute de esta actividad inusual (Comercio, 2015).

Se entregará la investigación al Gobierno Autónomo Descentralizado del Cantón Cevallos para motivar a los emprendedores locales realicen sus nuevos negocios con bases fortalecidas en el diseño planificado para ofrecer agroturismo y comercializar en base a capacitación, asistencia técnica y apoyo de las entidades financieras para el inicio y desarrollo del negocio (GADMCC, 2013).

La educación a la comunidad es importante para aprovechar la infraestructura y la participación de los sectores productivos como: calzado, agricultura orgánica, frutícola, apícola, avícola, ganadero, gastronomía, turístico pecuario, entre otras; éstos se integrarán de manera ordenada y planificada para no ejecutar esfuerzos aislados y obtener un posicionamiento diferenciado por tratarse de un lugar estratégico por las características climáticas y por formar parte del circuito del Tren Crucero Interandino.

\section{CONCLUSIONES}

- Se entregará la investigación al Gobierno Autónomo Descentralizado del Cantón Cevallos para motivar a los emprendedores locales realicen sus nuevos negocios con bases fortalecidas en el diseño planificado para ofrecer agroturismo y comercializar en base a capacitación, asistencia técnica y apoyo de las entidades financieras para el inicio y desarrollo del negocio.

- La educación a la comunidad es importante del emprendimiento en agroturismo y los beneficios que brindan los paisajes cultivados y aprovechar la infraestructura y la participación de los sectores productivos como: calzado, agricultura orgánica, frutícola, apícola, avícola, ganadero, gastronomía, turístico pecuario, entre otras.

- Aprovechar e integrar de manera ordenada y planificada para no ejecutar esfuerzos aislados y obtener un posicionamiento diferenciado por tratarse de un lugar estratégico por las características climáticas y por formar parte del circuito del Tren Crucero Interandino.

RECEIVED: April, 2017

REVISED: August, 2017

Referencia Bibliográficas

Alburquerque, F. (s/d de Abril de 2004). Desarrollo económico local. Obtenido de http://repositorio.cepal.org/bitstream/handle/11362/10946/082157171_es.pdf?sequence=1\& isAllowed $=\mathrm{y}$

Alvarez, M. (30 de Noviembre de 2015). Diseño de circuitos turísticos para la Empresa Pública Ferrocarriles del Ecuador en la ruta patrimonial del tren, tramo Riobamba-Huigra, provincia de Chimborazo. Obtenido de http://dspace.espoch.edu.ec/handle/123456789/4251 
Cevallos, R. G. (3 de Julio de 2015). IDENTIFICACIÓN PROSPECTIVA DE COMPONENTES EN EL PROCESO DE GESTIÓN AMBIENTAL DEL CANTÓN LA CONCORDIA, ECUADOR. Obtenido de https://www.researchgate.net/profile/Gabriel_Cevallos_Uve/publication/309458004_Identif icacion_prospectiva_de_componentes_en_el_proceso_de_gestion_ambiental_del_Canton_ La_Concordia_Ecuador/links/58111df108ae009606be7d98.pdf

Chiriboga, C. y. (s/d de Mayo de 2011). AGENDAS PARA LA TRANSOFRMACIÓN PRODUCTIVA TERRITORIAL: PROVINCIA DE TUNGURAHUA. Obtenido de http://www.produccion.gob.ec/wp-content/uploads/downloads/2013/02/AGENDATERRITORIAL-TUNGURAHUA.pdf

Comercio, E. (27 de Marzo de 2015). Tungurahua espera recuperar la actividad frutícola. Obtenido de http://www.elcomercio.com/actualidad/tungurahua-espera-recuperar-actividadfruticola.html

Crosby, A. (s/d de Febrero de 2009). Re-inventando el turismo rural. Obtenido de https://books.google.es/books?hl=es\&lr=\&id=wr4RzwV0GycC\&oi=fnd\&pg=PA13\&dq=Si +la+oferta+de+agroturismo+atrae+a+los+turistas+aumentar\%C3\% A 1+los+ingresos+de+la +poblaci\% $3 \% \mathrm{~B} 3 \mathrm{n}+\mathrm{rural}+$ siempre+conservando+la+realidad+natural.+Adem $\% \mathrm{C} 3 \% \mathrm{~A} 1 \mathrm{~s},+$ se+++proteger\%C3\%A1+el+

Exterior, D. N. (1 de Febrero de 1999). LEY ORGÁNICA DE LA LIBRE LEY ORGÁNICA DE LA LIBRE LEY ORGÁNICA DE LA LIBRE LEY ORGÁNICA DE LA LIBRE COMPETENCIA ECONOMICA . Obtenido de http://www.oecd.org/daf/competition/prosecutionandlawenforcement/38858121.pdf

GADMCC. (9 de Diciembre de 2013). LA ORDENANZA QUE DETERMINA LA ESTRUCTURA ORGANICO FUNCIONAL POR PROCESOS DEL GOB!ERNO AUTONOMO DESCENTRALIZADO MUNICIPAL DEL CANTON CEVALLOS. Obtenido de file:///C:/Users/Eufemia\%20Ramos/Downloads/Ordenanza\%20que\%20Determina\%20la\%2 0Estructura\%20Organico\%20Funcional\%20por\%20Procesos\%20del\%20Gobierno\%20Aut onomo\%20Descentralizado\%20Municipal\%20del\%20canton\%20.pdf

Ganán, R. (s/d de Noviembre de 2015). "EL TREN TURÍSTICO Y SU INCIDENCIA ECONÓMICA EN EL CANTÓN DURÁN, PARROQUIA ELOY ALFARO, PROVINCIA DEL GUAYAS: PERIODO 2012-2014". Obtenido de http://repositorio.ug.edu.ec/bitstream/redug/9195/1/Tren\%20Turistico\%20y\%20su\%20inci dencia\%20Economica\%20en\%20Duran\%202012-2014.pdf

General, A. (19-24 de Octubre de 2003). Día Mundial del Turismo: Información sobre las actividades de los años 2002, 2003, y adopción de los lemas y designación de los paises anfitriones de su celebración en 2004 y 2005. Obtenido de http://www.eunwto.org/doi/abs/10.18111/unwtogad.2003.3.u25105p2178325mv?journalCode=unwtoga d 
Inostroza, G. (s/d de Enero de 2008). APORTES PARA UN MODELO DE GESTIÓN SOSTENIBLE DEL TURISMO . Obtenido de http://mingaonline.uach.cl/pdf/gestur/n10/art06.pdf

José, S. (28 de Octubre de 2003). DECLARACIÓN DE SAN JOSÉ SOBRE TURISMO RURAL COMUNITARIO. Obtenido de file://C:/Users/Eufemia\%20Ramos/Downloads/E0200011\%20(1).pdf

Marreno, T. y. (s/d de s/m de 2014). Algunas Consideraciones Sobre La Medición De La Competitividad De DestinosTurísticos. Obtenido de http://retos.mes.edu.cu/index.php/retojs/article/view/60

Montaner, C. y. (s/d de s/m de 1998). Diccionario de turismo. Obtenido de https://books.google.es/books/about/Diccionario_de_turismo.html?hl=es\&id=Ad6JOwAAC AAJ

Normativa, D. d. (s/d de s/m de 2002). Ley de Turismo. Obtenido de http://www.innovaempresarial.com.ec/wp-content/uploads/2016/05/LEY-DETURISMO.pdf

Pilar, P. y. (s/d de s/m de 2011). El incumplimiento de las obligaciones tributarias de predio rural en el gobierno autónomo descentralizado municipal de Tisaleo, y su incidencia en la aplicación de mecanismos de cobranza legales año 2011. Obtenido de http://repositorio.uta.edu.ec/handle/123456789/1844

Recalde, R. (s/d de Octubre de 2010). Turismo rural sustentable y capital social: el caso del Proyecto Chachimbiro. Obtenido de http://67.192.84.248:8080/handle/10469/7619\#.WKXF94-cHIU

Rosario, L. y. (s/d de s/m de 2011). La producción de calzado en el cantón Cevallos, provincia de Tungurahua y su impacto en el empleo. Obtenido de http://repositorio.uta.edu.ec/handle/123456789/1961

UTA. (s/d de Octubre de 2016). Desarrollo Regional en Tungurahua. Obtenido de http://www.agenda2035.ec/sites/default/files/UTA\%20DESARROLLO\%20REGIONAL.pd $\mathrm{f}$

Valenzuela, L. (28 de Noviembre de 2007). ESTRATEGIAS E INSTRUMENTOS PARA EL DESARROLLO SOSTENIBLE DEL TURISMO RURAL . Obtenido de http://glifos.unis.edu.gt/digital/tesis/2007/21679.pdf

Zamora, M. (s/d de s/m de 2016). Diseño de un producto de agroturismo para el cantón Cevallos, provincia de Tungurahua. Obtenido de http://dspace.espoch.edu.ec/handle/123456789/5173 


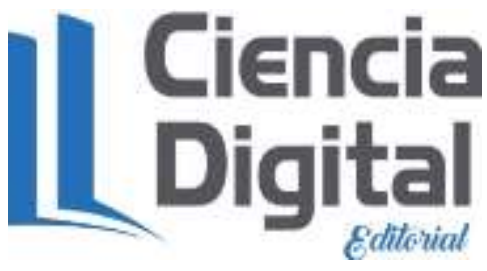

El artículo que se publica es de exclusiva responsabilidad de los autores y no necesariamente reflejan el pensamiento de la Revista Ciencia Digital.

El articulo queda en propiedad de la revista y, por tanto, su publicación parcial y/o total en otro medio tiene que ser autorizado por el director de la Revista Ciencia 